UDC 517.9

W. I. Skrypnik (Inst. Math. Nat. Acad. Sci. Ukraine, Kyiv)

\title{
LONG-RANGE ORDER IN GIBBS LATTICE CLASSICAL LINEAR OSCILLATOR SYSTEMS
}

\section{ДАЛЕКИЙ ПОРЯДОК У ГІББСІВСЬКИХ ГРАТКОВИХ КЛАСИЧНИХ СИСТЕМАХ ЛІНІЙНИХ ОСЦИЛЯТОРІВ}

Existence of the ferromagnetic long range order (lro) is proven for Gibbs classical lattice systems of linear oscillators interacting via a strong polynomial pair nearest neighbor (n-n) ferromagnetic potential and other (nonpair) potentials which are weak if they are not ferromagnetic. A generalized Peierls argument and two different contour bounds are our main tools.

Доведено існування феромагнітного далекого порядку для гіббсівської класичної граткової системи лінійних осциляторів, що взаємодіють завдяки сильному парному поліноміальному феромагнітному потенціалу близьких сусідів та іншим (непарним) потенціалам, які слабкі, якщо не феромагнітні. При цьому використано узагальнений аргумент Пайєрлса та дві контурні нерівності.

1. Introduction and main result. Let's consider Gibbs classical and quantum systems of one-dimensional oscillators on the $d$-dimensional hypercubic lattice $\mathbb{Z}^{d}$, with the potential energy $U\left(q_{\Lambda}\right)=U\left(-q_{\Lambda}\right)$ on a set $\Lambda$ with the finite cardinality $|\Lambda|$, where $q_{\Lambda}$ is an array of $\left(q_{x}, x \in \Lambda\right), q_{x}$ is the oscillator coordinate taking value in $\mathbb{R}$. The potential energy is assumed to be a growing function at infinity. It is invariant under the simplest discrete symmetry, namely $\mathbb{Z}_{2}$, which is realized as a transformation of changing of all the signs of the oscillator variables. We tacitly assume, also, that the correlation functions exist in the thermodynamic limit.

Let \langle\rangle$_{\Lambda}$ denote the Gibbs average for the system confined to $\Lambda$ (see (2.1)) and

$$
\chi_{x}^{+}\left(q_{\Lambda}\right)=\chi_{(0, \infty)}\left(q_{x}\right), \quad \chi_{x}^{-}\left(q_{\Lambda}\right)=\chi_{(-\infty, 0)}\left(q_{x}\right), \quad \sigma_{x}\left(q_{\Lambda}\right)=q_{x}, \quad s_{x}=\operatorname{sign} \sigma_{x}
$$

where $\chi_{(a, b)}$ is the characteristic function of the open interval $(a, b)$. Taking into account that $\chi_{x}^{+(-)}=\frac{1}{2}\left[1 \pm s_{x}\right]$ one obtains

$$
4\left\langle\chi_{x}^{+} \chi_{y}^{-}\right\rangle_{\Lambda}=1+\left\langle s_{x}\right\rangle_{\Lambda}-\left\langle s_{y}\right\rangle_{\Lambda}-\left\langle s_{x} s_{y}\right\rangle_{\Lambda}
$$

Since the systems are invariant under the transformation of changing signs of the oscillator variables the third and the second terms in the right-hand side of last equality are equal to zero and

$$
\left\langle s_{x} s_{y}\right\rangle_{\Lambda}=1-4\left\langle\chi_{x}^{+} \chi_{y}^{-}\right\rangle_{\Lambda}
$$

Hence if

$$
\left\langle\chi_{x}^{+} \chi_{y}^{-}\right\rangle_{\Lambda}<\frac{1}{4}
$$

then the ferromagnetic lro for the unit spins (unit spin lro) $s_{x}$ occurs, i.e.,

$$
\left\langle s_{x} s_{y}\right\rangle_{\Lambda} \geq a>0
$$

where $a$ is independent of $\Lambda$. (1.1) may be derived from the generalized Peierls principle.

Theorem 1.1. Let the contour bound holds

$$
\left\langle\prod_{\langle x, y\rangle \in \Gamma} \chi_{x}^{+} \chi_{y}^{-}\right\rangle_{\Lambda} \leq e^{-|\Gamma| E}
$$


where $\Gamma$ is the set of pairs of nearest neighbors and $e^{-2 E}<\frac{1}{6}$. Then there exists a constant a independent $\Lambda$ such that

$$
\left\langle\chi_{x}^{+} \chi_{y}^{-}\right\rangle_{\Lambda} \leq a e^{-2 d E}
$$

Corollary 1.1. If

$$
e^{-2 E}<\min \left(6^{-1},\left(4 a^{\frac{1}{d}}\right)^{-1}\right)
$$

then the unit spin ferromagnetic lro occurs, that is (1.2) holds.

The first application of the Peierls argument for proving of the existence of the unit spin lro was given in [1] for Euclidean two-dimensional quantum boson field system which can be approximated by a lattice linear oscillator system (the analog of $s_{x}$ was a smeared over unit cubes, centered at the sites of the square lattice, boson field). A wide range of applicability of the argument was demonstrated later in [2] for anisotropic Heisenberg systems and simplest classical systems of many component oscillators interacting via a pair n-n potential (see Remark 1). In [3-6] we extended the range to lattice linear oscillator systems with a strong ferromagnetic $n-n$ belinear interaction and nonequilibrium simplest lattice systems of interacting Brownian linear oscillators, respectively.

In this paper, we prove the contour bound (1.3), infinite growth of $E$ in the parameter $g$, and existence of the lro for two lattice linear oscillator systems with the following potential energy ( $\Lambda$ is a hypercube with cardinality $|\Lambda|$ ) which is the most general in the class of energies with polynomial external fields and pair n-n potentials

$$
U\left(q_{\Lambda}\right)=\sum_{x \in \Lambda}\left(u_{0}\left(q_{x}\right)-g \eta_{0} q_{x}^{2 n_{0}}\right)+\sum_{\langle x, y\rangle \in \Lambda} \phi\left(q_{x}, q_{y}\right)+U^{\prime}\left(q_{\Lambda}\right), \quad \eta_{0}=0,1,
$$

where $U^{\prime}$ equals zero for coinciding arguments, $\langle x, y\rangle$ means nearest neighbors, $u$ is a bounded below polynomial of the $2 n$-th degree $n>n_{0}$,

$$
u_{0}(q)=\eta q^{2 n}+u^{1}(q), \quad u^{1}(q)=\sum_{j=1}^{n-1} \eta_{j} q^{2 j}
$$

$\phi$ is given either by

$$
\eta_{0}=0, \quad \phi\left(q_{x}, q_{y}\right)=-g\left(q_{x}^{k} q_{y}^{l}+q_{x}^{l} q_{y}^{k}\right), \quad k+l=2 n_{0},
$$

or

$$
\eta_{0}=1, \quad \phi\left(q_{x}, q_{y}\right)=g_{0}\left(q_{x}-q_{y}\right)^{2 n_{1}} Q^{\prime}\left(q_{x}, q_{y}\right), \quad g_{0}=g^{\frac{\xi}{2\left(n-n_{0}\right)}}=z^{-\xi},
$$

$k, l$ are odd positive numbers, $Q^{\prime}$ is a positive symmetric even homogeneous polynomial of the $2 n_{2}$-th degree, i.e., is the sum of the terms $q^{s} q^{m}, s+m=2 n_{2}$ with positive coefficients, $n_{1}+n_{2}<n$, such that $Q^{\prime}(1,1)=1$ and $n_{2}^{\prime}=\max (s, m)$; for $\eta_{0}=0$ the function $U^{\prime}$ is such that the Gibbs averages satisfy the GKS inequality [7, 8]. Such the systems we'll call GKS-type systems (see Remark 2). Prime will not mean differentiation in our notations.

The parameter $g$ determines depthes of minima of the potential $u(q)=u_{0}(q)-$ $-g \eta_{0} q^{2 n_{0}}$ (see Remark 3).

It will be proven in Appendix $\mathrm{C}$ the following important equality: 


$$
q_{x}^{k} q_{y}^{l}+q_{x}^{l} q_{y}^{k}=q_{x}^{2 n_{0}}+q_{y}^{2 n_{0}}-\left(q_{x}-q_{y}\right)^{2} Q\left(q_{x}, q_{y}\right),
$$

where $Q$ is a positive homogeneous polynomial with the degree $2\left(n_{0}-1\right)$ (it is a sum of $Q^{\prime}$ and a positive homogeneous noneven polynomial). This equality transforms the case $\eta_{0}=0$ into the case $\eta_{0}=2 d$. By rescaling of variables the case $\eta_{0}>0$ may be mapped into the case $\eta_{0}=1$.

Our first result, generalizing the result of [6], is formulated as the following theorem.

Theorem 1.2. For classical oscillator GKS-type systems with the potential energies (1.4) with $\eta_{0}=0$ the contour bound (1.3) holds with the positive function $E$ in $g$, which is growing at infinity as $g^{\frac{n}{n-n_{0}}}$, and the unit spin lro occurs at sufficiently large $g$.

This theorem follows from the explicit expression for $E$ in (1.3) given by (2.2) - (2.4) and Theorem 2.1.

Since the corner stone of our technique for $\eta_{0}=1$ is the generalized Ruelle superstability bound we have to introduce the superstability and regularity conditions for the rescaled potential energy. We rescale all the variables by $z^{n-1}$ and put $u_{g}(q)=u\left(z^{n-1} q\right)$, $\phi_{g}\left(q, q^{\prime}\right)=\phi\left(z^{n-1} q, z^{n-1} q^{\prime}\right)$,

$$
\begin{aligned}
& u_{*}(q)=u_{g}\left(q+e_{0}\right)-u_{g}\left(e_{0}\right), \quad \phi_{*}\left(q, q^{\prime}\right)=\phi_{g}\left(q+e_{0}, q^{\prime}+e_{0}\right), \\
& U_{g}\left(q_{\Lambda}\right)=U\left(z^{n-1} q_{\Lambda}\right), \quad U_{*}\left(q_{X}\right)=U_{g}\left(q_{X}+e_{0}\right)-|X| u_{g}\left(e_{0}\right),
\end{aligned}
$$

where $e_{0}$ is the deepest minimum of $u_{g}$ which grows in $g$ at infinity (see Section 3 ), $e_{0}$ is easily found for the potential $u_{0}=\eta q^{2 n}$ leading to the effective potential $u$ which has only two symmetric wells. One may see that $U_{*}$ has a finite limit when $g$ tends to infinity. This fact plays an important role in our method since we'll obtain bounds for the correlation functions generated by $U_{*}$.

Let's put

$$
W_{*}\left(q_{X} ; q_{Y}\right)=U_{*}\left(q_{\Lambda}\right)-U_{*}\left(q_{Y}\right)-U_{*}\left(q_{X}\right), \quad \Lambda=X \cup Y .
$$

The same relation will hold, also, for $U_{*}^{\prime}$ and $W_{*}^{\prime}$.

We require validity of the following superstability and regularity conditions:

$$
\begin{gathered}
U_{*}\left(q_{\Lambda}\right) \geq \sum_{x \in \Lambda} u_{*}^{-}\left(q_{x}\right), \quad u_{*}^{-}(q)=u_{*}(q)-\zeta v^{0}\left(z^{n} q\right)-\zeta_{0}, \\
\left|W_{*}^{\prime}\left(q_{X} ; q_{Y}\right)\right| \leq \sum_{x \in X, y \in Y} \Psi^{\prime}(|x-y|)\left(v^{0}\left(q_{x}\right)+v^{0}\left(q_{y}\right)\right), \\
v^{0}(q)=\sum_{j=1}^{n-1} q^{2 j},
\end{gathered}
$$

where the positive $L^{1}$-function $\Psi^{\prime}$, and numbers $\zeta, \zeta_{0} \geq 0$ do not depend on $g$. The second term in the expression for $u_{*}^{-}$is a contribution of the negative (nonferromagnetic) term in $U^{\prime}$ which shows that the latter is always small, that is, it depends on positive powers of $g^{-1}$. Condition (1.6) can be substituted by the inequality for $U$ coinciding with (1.6) in which $v^{0}(z q)$ is inserted instead of $v^{0}\left(z^{n} q\right)$. Conditions (1.6), (1.7) allow positive (ferromagnetic) interaction terms in $U$ to be large. Interaction is stronger for translation invariant potentials. For translation invariant interaction two conditions for the functions $U^{\prime}, W^{\prime}$ as in [3,4] may be postulated in such the way that they will imply (1.6), (1.7), that is (1.6), (1.7) generalize the superstability and regularity conditions from [3, 4]. 
Theorem 1.3. Let the potential energy of the classical oscillator systems in (1.4) with $\eta_{0}=1$ satisfy (1.6), (1.7) with $\Psi^{\prime}$ having a finite range and

$$
n-2\left(n_{1}+n_{2}\right)<\xi \leq 2(n-1) n_{1}-2 n_{2} .
$$

Then the contour bound (1.3) holds with the positive function $E$ in $g$, which is growing at infinity as $g^{\frac{n}{2\left(n-n_{0}\right)}}$, and the unit spin lro occur at sufficiently large $g$.

This theorem is based on the derivation of the $E$ from (1.3) in the form

$$
E=\beta e_{0}-e_{*}(g)+2 \ln I_{*}\left(g, Q^{0}\right),
$$

where the integral $I_{*}\left(g, Q^{0}\right)$ is given by (2.13) and $e_{*}(g)$ is extracted from our generalization of the Ruelle superstability bound proposed in Theorem 2.2. The corner stone of its proof is check of the condition that the second and third terms have to grow at infinity in $g$ not so fast as $e_{0}$.

These theorems establish existence of phase transitions for the classical systems with pair short-range Kunz potentials since in the high-temperature phase there is a decrease of correlations [9]. They imply that the behavior of the strength of the $n-n$ pair interaction has to be correlated with the depth of the deepest minimum of the effective potential and a character of the potential itself (this is done through the formula (2.8)).

The right-hand side of (1.8) guarantee that $u_{g}$ is a bounded function in $g$ and that $W_{*}$ satisfies (1.7) with $\Psi(|x-y|)=\Psi^{\prime}(|x-y|)+\tilde{C} \delta_{|x-y|, 1}$ instead of $\Psi_{|x-y|}^{\prime}$, where $\delta_{x, y}$ is the Kronecker symbol (see Appendix A). The left-hand side of (1.8) arises from application of the exponential bound (2.8) for the product of the characteristic functions in (1.3) and the necessity of suppression of the first unbounded term in the expression $Q_{g}$ in it $\left(Q_{g}\right.$ in (2.10) does not allow the strength of the nn ferromagnetic interaction $g_{0}$ be arbitrary small). As a result of this bound asymptotics of $\beta^{-1} E$ in $g$ coincides with the asymptotics of the deepest minimum of the external potential $u$ in the last theorem.

Theorem 1.3 generalizes our previous results in $[3,4]$ for finite-range interactions with the help of the new version of the superstability bound that has appeared already in [5]. It was applied in [5] for a proof of lro in nonequilibrium systems of Brownian oscillators with Gibbsian initial states and it contains an exponent with a potential energy, generated by the pair ferromagnetic n-n potential, before the right-hand side of the usual superstability bound.

The main ideas of our method are exposed in the next section where it is shown how Theorems 2.1, 2.2 allow to prove Theorems 1.2, 1.3. In Section 3 we formulate and prove Lemma 3.1 which gives bounds necessary for proofs of Theorems 2.1, 2.2. In Section 4 our basic superstability bound is presented which implies validity of the superstability bounds in Theorem 2.2, Lemma 4.1 and Corollary 4.2 establish asymptotics of $e_{*}$ from Theorem 2.2. Proof of Theorem 1.1 and Theorem 4.1 may be found in [5].

Development of our approach demanded a clarification of the question in what way the Ruelle superstability bound [10] depends on the behavior of the function $\Psi$ from at infinity in the regularity condition (4.2). It turned out that the usual superstability bound does not feel such the behavior. We introduce the condition

$$
\sum_{x}|x|^{k} \Psi(|x|)<\infty
$$

which coincide with the Ruelle condition for $k=1$ and establish the asymptotics of the main constant $c$ of the new superstability bound in an arbitrary small constant $\varepsilon$ in 
(4.3) in terms of $k$ (see Corollary 4.1). For finite-range interactions $c_{0}$ grows very slow at zero in $\varepsilon$ and this helps to find asymptotics of $E$ in (1.9) after putting $\varepsilon=z^{2(n-1) n}$. A generalization of Theorem 1.3 to infinite-range interaction demands a more profound change of the Ruelle arguments which concern asymptotics of $c^{0}$ in $\varepsilon$.

We expect that that our results may be generalized to the potentials $u \exp \{\tilde{u}\}$, where $u, \tilde{u}$ are polynomials, for which $e_{0}$ is easily found.

2. Contour bounds. For classical linear oscillator systems with the potential energy $U$ and the inverse temperature $\beta$ the Gibbs average is given by

$$
\begin{gathered}
\left\langle F_{X}\right\rangle_{\Lambda}=Z_{\Lambda}^{-1} \int F_{X}\left(q_{X}\right) e^{-\beta U\left(q_{\Lambda}\right)} d q_{\Lambda}=\int F_{X}\left(q_{X}\right) \rho^{\Lambda}\left(q_{X}\right) d q_{X}, \\
\rho^{\Lambda}\left(q_{X}\right)=Z_{\Lambda}^{-1} \int e^{-\beta U\left(q_{\Lambda}\right)} d q_{\Lambda \backslash X}, \quad Z_{\Lambda}=\int e^{-\beta U\left(q_{\Lambda}\right)} d q_{\Lambda} .
\end{gathered}
$$

Here the integrations are performed over $R^{|\Lambda|}, R^{|X|}, R^{|\Lambda \backslash X|}$ and $\rho^{\Lambda}$ are the correlation functions, where $|X|$ is the cardinality of $X$.

The contour bound for $\eta_{0}=1$ is derived from the new generalized Ruelle superstability bound for correlation functions and reduced density matrices. The contour bound for $\eta_{0}=0$ is obtained with a help of a generalized Bricmont - Fontaine argument [11] and results from the inequality for $k=2 s+1, l=2\left(n_{0}-s\right)-1$

$$
\chi_{x}^{+} \chi_{y}^{-} \leq e^{-\frac{g \beta}{2}\left(\sigma_{x}^{k} \sigma_{y}^{l}+\sigma_{x}^{l} \sigma_{y}^{k}\right)}
$$

Its proof is easy

$$
\begin{gathered}
\chi_{x}^{+} \chi_{y}^{-}=e^{-\frac{g \beta}{2}\left(\sigma_{x}^{k} \sigma_{y}^{l}+\sigma_{x}^{l} \sigma_{y}^{k}\right)} e^{\frac{g \beta}{2}\left(\sigma_{x}^{k} \sigma_{y}^{l}+\sigma_{x}^{l} \sigma_{y}^{k}\right)} \chi_{x}^{+} \chi_{y}^{-} \leq \\
\leq e^{-\frac{g \beta}{2}\left(\sigma_{x}^{k} \sigma_{y}^{l}+\sigma_{x}^{l} \sigma_{y}^{k}\right)} \chi_{x}^{+} \chi_{y}^{-} \leq e^{-\frac{g \beta}{2}\left(\sigma_{x}^{k} \sigma_{y}^{l}+\sigma_{x}^{l} \sigma_{y}^{k}\right)} .
\end{gathered}
$$

Here one takes into account that $\sigma_{x} \geq 0, \sigma_{y} \leq 0$. The last inequality is employed for the proof of the contour bound as follows:

$$
\begin{aligned}
& \left\langle\prod_{\langle x, y\rangle \in \Gamma} \chi_{x}^{+} \chi_{y}^{-}\right\rangle_{\Lambda} \leq\left\langle e^{-\frac{g \beta}{2} \sum}\langle x, y\rangle \in \Gamma\left(\sigma_{x}^{k} \sigma_{y}^{l}+\sigma_{x}^{l} \sigma_{y}^{k}\right)\right\rangle_{\Lambda}= \\
& =\left\langle e^{\frac{g \beta}{2} \sum}\langle x, y\rangle \in \Gamma\left(\sigma_{x}^{k} \sigma_{y}^{l}+\sigma_{x}^{l} \sigma_{y}^{k}\right)\right\rangle_{\Lambda[\Gamma]} \leq \\
& \left.\leq e^{-\frac{g \beta}{2} \sum}\langle x, y\rangle \in \Gamma\left\langle\sigma_{x}^{k} \sigma_{y}^{l}+\sigma_{y}^{k} \sigma_{y}^{l}\right)\right\rangle_{\Lambda[\Gamma]}=e^{E_{\Gamma}},
\end{aligned}
$$

where $\langle., .\rangle_{\Lambda[\Gamma]}$ is the average corresponding to the potential energy

$$
U_{\Gamma}\left(q_{\Lambda}\right)=U\left(q_{\Lambda}\right)+\frac{g}{2} \sum_{\langle x, y\rangle \in \Gamma}\left(q_{x}^{k} q_{y}^{l}+q_{x}^{l} q_{y}^{k}\right) .
$$

In last line, we applied the Jensen inequality. Taking into account that the Gibbs average is a monotone increasing function in $J_{\left(A, n_{A}\right)}$ (a result of the GKS inequality, see Remark 2) we obtain

$$
E_{\Gamma} \geq E|\Gamma|, \quad E=\beta g\left\langle\sigma^{k}{\sigma^{\prime}}^{l}+\sigma^{l}{\sigma^{\prime}}^{k}\right\rangle
$$


where

$$
\begin{gathered}
\left\langle\sigma^{k}{\sigma^{\prime}}^{l}+\sigma^{l}{\sigma^{\prime}}^{k}\right\rangle=Z^{-1}(2) \int\left(q_{1}^{k} q_{2}^{l}+q_{1}^{l} q_{2}^{k}\right) e^{-\beta u\left(q_{1}, q_{2}\right)} d q_{1} d q_{2} \\
Z(2)=\int e^{-\beta u\left(q_{1}, q_{2}\right)} d q_{1} d q_{2} \\
u\left(q_{1}, q_{2}\right)=u_{0}\left(q_{1}\right)+u_{0}\left(q_{2}\right)-g\left(q_{1}^{k} q_{2}^{l}+q_{1}^{l} q_{2}^{k}\right),
\end{gathered}
$$

$k, l$ are positive integers, $k=2 s-1, l=2\left(n_{0}-s\right)-1$, i.e., $k+l=2 n_{0}$ and $\beta$ is the inverse temperature. Formula (1.3) together with (2.4) is the generalized BF contour bound.

The asymptotics of $E$ in (2.2) is determined with the help of the following theorem.

Theorem 2.1. There exists a positive function in $\beta \quad C_{+}$independent of $g$ and $a$ positive constant $c_{-}$such that for sufficiently large $g$ two following inequalities hold:

$$
\begin{aligned}
& \left\langle\sigma^{2 n_{0}}+\sigma^{\prime 2 n_{0}}\right\rangle \geq C_{+} g^{-1} g^{\frac{n}{n-n_{0}}},
\end{aligned}
$$

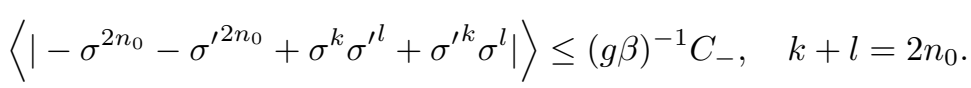

The most important implication of this theorem is the following statement.

Corollary 2.1. Let E be given by (2.2). Then E grows in $g$ at infinity as $g^{\frac{n}{n-n_{0}}}$.

This corollary is a result of (2.5), (2.6) and the obvious inequality

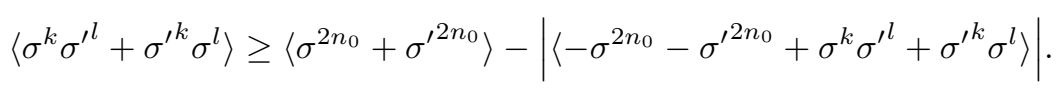

Theorem 1.2 is proved.

Main idea of proof of Theorem 1.3. Let's put

$$
\rho_{*}^{\Lambda}\left(q_{X}\right)=\rho_{g}^{\Lambda}\left(q_{X}+e_{0}\right) .
$$

From the translation invariant character of the Lebesque measure it follows that $\rho_{*}^{\Lambda}$ is expressed in terms of $U_{*}$. The dependence of $u_{*}$, superstability and regularity conditions on $g$ enable us to prove Theorem 1.3 with the help of the following theorem.

Theorem 2.2. Let the conditions of Theorem 1.3 be satisfied. Then for the correlation functions $\rho_{*}^{\Lambda}$ the following supertability bound is valid:

$$
\rho_{*}^{\Lambda}\left(q_{X}\right) \leq \exp \left\{|X| e_{*}(g)-\beta\left[U_{*}^{+}\left(q_{X}\right)+\sum_{x \in X} u_{*}^{+}\left(q_{x}\right)\right]\right\},
$$

where

$$
u_{*}^{+}=u_{*}^{-}-3 \epsilon v^{0}, \quad \epsilon=z^{2 n(n-1)},
$$

$e_{*}(g)$ depends neither on oscillator variables nor $\Lambda, U_{*}^{+}$is the positive part of $U_{*}$ generated by a pair potential and for arbitrary $\delta>0$ and sufficiently large $g$

$$
\delta e_{0} \geq e_{*}(g) .
$$

Inequality (2.7) differs from the usual superstability bound from [10] by the presence of $U_{*}^{+}$in its right-hand side. This term plays a significant role in proving (1.1) with positive $E(g)$ increasing at infinity in $g$ : it forbids the strength $g_{0}$ of the positive n-n pair 
interaction uncontrollably tend to zero after applying (2.8). Calculation of asymptotics of $e_{*}(g)$ is quite a nontrivial task that can be solved only with the help of the generalization of the Ruelle superstability bound proposed in Theorem 4.1 ((4.4) determines the structure of the main constant $c_{0}$ in the new superstability bound).

The exponential term appears in the bounds of the correlation functions of classical oscillator systems in the high-temperature phase if one applies the technique of the Kirkwood-Saltsburg relations considered in $[9,12]$. The similar term is present in the superstability bound in [13] for classical oscillator ferromagnetic systems with a special pair potential.

We derive the contour bound (1.3) for the potential energy (1.4) for $\eta_{0}=1$ with the help of (2.7) and the following bound which is a generalization of the similar bound from [3-5]:

$$
\prod_{\left\langle x, x^{\prime}\right\rangle \in \Gamma} \chi^{+}\left(q_{x}\right) \chi^{-}\left(q_{x^{\prime}}\right) \leq \exp \left\{-\beta\left[e_{0}|\Gamma|-Q_{g, \Gamma}\left(q_{\Lambda}\right)\right]\right\}
$$

where

$$
\begin{gathered}
Q_{g, \Gamma}\left(q_{\Lambda}\right)=\sum_{\left\langle x, x^{\prime}\right\rangle \in \Gamma} Q_{g}\left(q_{x}, q_{y}\right), \\
Q_{g}\left(q_{x}, q_{y}\right)=e_{0}^{-2\left(n_{1}+n_{2}\right)+1} \times \\
\times\left[2^{2 n_{2}} g_{0}^{-1} \phi\left(q_{x}, q_{y}\right)+\left(\frac{4}{3}\right)^{n_{1}+n_{2}}\left(\left|q_{x}^{2}-e_{0}^{2}\right|^{n_{1}+n_{2}}+\left|q_{y}^{2}-e_{0}^{2}\right|^{n_{1}+n_{2}}\right)\right] .
\end{gathered}
$$

The idea to apply the analogue of (2.8) goes back to [1]. (2.8) is a consequence of the following inequality:

$$
\chi^{+}\left(q_{x}\right) \chi^{-}\left(q_{y}\right) \leq e^{c\left[-R^{2\left(n_{1}+n_{2}\right)}+Q\left(q_{x}, q_{y} \mid R\right)\right]}, \quad R, c>0,
$$

where

$$
Q\left(q_{x}, q_{y} \mid R\right)=2^{2 n_{2}} g_{0}^{-1} \phi\left(q_{x}, q_{y}\right)+\left(\frac{4}{3}\right)^{n_{1}+n_{2}}\left(\left|q_{x}^{2}-R^{2}\right|^{n_{1}+n_{2}}+\left|q_{y}^{2}-R^{2}\right|^{n_{1}+n_{2}}\right) .
$$

It is derived easily from two inequalities

$$
\begin{gathered}
\chi^{+}\left(q_{x}\right) \chi^{-}\left(q_{y}\right) \leq e^{\left.c\left[-R^{2\left(n_{1}+n_{2}\right)}+g_{0}^{-1} 2^{2 n_{2}} \phi\left(q_{x}, q_{y}\right)\right)\right]}, \quad\left|q_{x}\right|,\left|q_{y}\right| \geq 2^{-1} R, \\
\chi^{+}\left(q_{x}\right) \chi^{-}\left(q_{y}\right) \leq e^{c\left[-R^{2\left(n_{1}+n_{2}\right)}+\left(\frac{4}{3}\right)^{n_{1}+n_{2}}\left(\left|q_{x}^{2}-R^{2}\right|^{n_{1}+n_{2}}+\left|q_{y}^{2}-R^{2}\right|^{n_{1}+n_{2}}\right)\right]}, \\
\left|q_{x}\right|,\left|q_{y}\right| \leq 2^{-1} R .
\end{gathered}
$$

To derive the first inequality one has to account the inequalities for $q_{x} \geq \frac{R}{2}, q_{y} \leq-\frac{R}{2}$

$$
\begin{gathered}
\left(q_{x}-q_{y}\right)^{2 n_{1}} \geq R^{2 n_{1}}, \\
Q^{\prime}\left(q_{x}, q_{y}\right)=Q^{\prime}\left(\left|q_{x}\right|,\left|q_{y}\right|\right) \geq Q^{\prime}\left(\frac{R}{2}, \frac{R}{2}\right)=\left(\frac{R}{2}\right)^{2 n_{2}} Q^{\prime}(1,1)=\left(\frac{R}{2}\right)^{2 n_{2}} .
\end{gathered}
$$

For $\left|q_{x}\right| \leq \frac{R}{2},\left|q_{y}\right| \geq \frac{R}{2}$ the second term in the expression for $Q$ is not less than $R^{2\left(n_{1}+n_{2}\right)}$. Inequality (2.8) results from (2.9) putting 


$$
Q_{g}\left(q, q^{\prime}\right)=Q\left(q, q^{\prime} \mid R\right), \quad c=\beta e_{0}^{-2\left(n_{1}+n_{2}\right)+1}, \quad R=e_{0} .
$$

Let's substitute (2.8) into (1.3), translate all the variables by $e_{0}$ in the Gibbs average and apply (2.7)

$$
\begin{gathered}
\left\langle\prod_{\langle x, y\rangle \in \Gamma} \chi_{x}^{+} \chi_{y}^{-}\right\rangle_{\Lambda} \leq e^{-\beta e_{0}|\Gamma|} \int \rho_{*}^{\Lambda}\left(q_{\Gamma}\right) \exp \left\{\beta Q_{g, \Gamma}\left(q_{\Gamma}\right)\right\} d q_{\Gamma} \leq \\
\leq e^{-\left(\beta e_{0}-e_{*}(g)\right)|\Gamma|} \int \exp \left\{-\beta\left[\sum_{x \in \Gamma} u_{*}^{+}\left(q_{x}\right)+\right.\right. \\
\left.+\sum_{\langle x, y\rangle \in \Gamma}\left(\phi_{g}\left(q_{x}+e_{0}, q_{y}+e_{0}\right)-Q_{g}\left(q_{x}+e_{0}, q_{y}+e_{0}\right)\right]\right\} d q_{\Gamma} .
\end{gathered}
$$

We'll show in the next section that $e_{0}=\mu z^{-n}$, where the $\mu$ is a bounded function of $g$. For $u_{0}(q)=\eta q^{2 n}$ this function is a constant. Now, if

$$
z^{-\xi} z^{2(n-1)\left(n_{1}+n_{2}\right)}>e_{0}^{-2\left(n_{1}+n_{2}\right)+1},
$$

or (we use the equality $\phi_{g}=z^{2\left(n_{1}+n_{2}\right)(n-1)} \phi$ )

$$
-\xi+2(n-1)\left(n_{1}+n_{2}\right)<\left(2\left(n_{1}+n_{2}\right)-1\right) n,
$$

then the terms with $\phi_{g}$ disappear in the right-hand side of (2.10) and we obtain for $I_{*}\left(g, Q^{0}\right)>1$

$$
\left\langle\prod_{\langle x, y\rangle \in \Gamma} \chi_{x}^{+} \chi_{y}^{-}\right\rangle_{\Lambda} \leq e^{-\left(\beta e_{0}-e_{*}(g)\right)|\Gamma|}\left(I_{*}\left(g, Q^{0}\right)\right)^{2|\Gamma|}
$$

where

$$
\begin{gathered}
I_{*}\left(g, Q^{0}\right)=\int e^{-\beta\left[u_{*}^{+}(q)-e_{0}^{-2\left(n_{1}+n_{2}\right)+1} Q^{0}\left(q ; e_{0}\right)\right]} d q, \\
Q^{0}\left(q ; e_{0}\right)=\left(\frac{4}{3}\right)^{n_{1}+n_{2}}\left|q\left(q+2 e_{0}\right)\right|^{n_{1}+n_{2}} .
\end{gathered}
$$

If $I_{*}\left(g, Q^{0}\right) \leq 1$ then (2.12) holds without the last term in its right-hand side. The condition $I_{*}\left(g, Q^{0}\right)>1$ is necessary in (2.12) to account the fact that in pairs from $\Gamma$ there may be coinciding sites. As a result (1.9) holds.

From Proposition 3.1 and (2.7a) it follows that $E$ in (1.9) grows at infinity in $g$ and the unit spin lro occurs in the system. This will conclude the proofs of Theorems 1.2, 1.3.

3. Asymptotics of integrals. Our main aim in this sections is to determine the asymptotics of $E$ in $g$ at infinity in formulas (1.9) and (2.2). For this purpose let's introduce the new potential $h$ and describe properties of the minima of $u_{g}$ and $u$ in terms of its minima

$$
u\left(z^{-1} q\right)=z^{-2 n} h(q), \quad u_{g}(q)=u\left(z^{n-1} q\right)=z^{-2 n} h\left(z^{n} q\right),
$$

where

$$
h(q)=\eta q^{2 n}-q^{2 n_{0}}+h^{1}(q), \quad h^{1}(q)=\sum_{j=1}^{n-1} \eta_{j} z^{2(n-j)} q^{2 j}=z^{2 n} u^{1}\left(z^{-1} q\right) .
$$


Let $e^{\prime}(j), \mu(j), e(j)$ be the minima of $u, h, u_{g}$, respectively. The deepest among the sequences will be denoted by $e_{+}, \mu, e_{0}$, respectively. Then

$$
e^{\prime}(j)=z^{-1} \mu(j), \quad e(j)=z^{-n} \mu(j) .
$$

It is so since for the equations for the minima we have

$$
\partial u\left(z^{-1} q\right)=\partial h(q)=0, \quad \partial u_{g}(q)=\partial h\left(z^{n} q\right)=0, \quad \partial=\frac{\partial}{\partial q} .
$$

$\mu$ is the only root of $\partial h$ which converges to the unique positive root $\mu_{0}$ of $\partial h^{0}$

$$
h^{0}(q)=\eta q^{2 n}-q^{2 n_{0}}, \quad \partial h^{0}(q)=2 q^{2 n_{0}-1}\left(\eta n q^{2\left(n-n_{0}\right)}-n_{0}\right)
$$

when $z$ tends to zero. It is simple and an analytical function of $z$ in the neighborhood of zero (see Section 1 in [14]), that is

$$
\mu=\mu^{0}+\sum_{k \geq 1} z^{k} \mu_{k}, \quad \mu^{0}=\left(\frac{n_{0}}{n \eta}\right)^{\frac{1}{2\left(n-n_{0}\right)}} .
$$

It is the deepest minimum of $h$ since other roots $\mu_{j}$ of $\partial h$ converge to zero for vanishing $z$. For these roots there is the following convergent expansion in the neighborhood of zero [14]:

$$
\mu(j)=\sum_{k \geq 1} z^{\frac{k}{l_{j}}} \mu_{k, j}, \quad \sum_{s} l_{s}=2 n_{0}-1, \quad 1 \leq l_{j} \leq 2 n_{0}-1 .
$$

Validity of these expansions can be confirmed for a special choice of $u^{1}$ (see Appen$\operatorname{dix} B$ ).

It is not difficult to check that

$$
\partial^{s} u_{g}(q)=z^{-2 n+s n} \partial^{s} h\left(z^{n} q\right), \quad \partial^{2} u_{g}(q)=\partial^{2} h\left(z^{n} q\right) .
$$

Relations (3.3), (3.4) yield

$$
\partial^{s} u_{g}(e(j))=z^{-2 n+s n} \partial^{s} h(\mu(j)), \quad \partial^{2} u_{g}(e(j))=\partial^{2} h(\mu(j)) .
$$

This implies $z$ the derivatives of $\partial^{s} u_{g}$ at the minima of $u_{g}$ tend to zero if $g$ tends to infinity and $s>2$. That is

$$
u_{g}\left(q+e_{0}\right)-u_{g}\left(e_{0}\right)=a q^{2}+p_{g}(q), \quad a=\partial^{2} h(\mu), \quad \lim _{q \rightarrow \infty} p_{g}(q)=0 .
$$

We'll restrict $z$ to the neighborhood of zero, i.e., the interval $\left[0, z^{0}\right]$, for which $z \leq 1$, $z^{n} \leq \frac{1}{4} \mu_{0}, \mu>\frac{1}{2} \mu^{0}, \mu_{j}<\frac{1}{2} \mu$. Let $\theta=\frac{1}{4} \min _{z \in\left[0, z^{0}\right]} \partial h^{2}(\mu) . \theta>0$ since $\partial h^{2}\left(\mu_{0}\right)>0$ and $\mu$ is continuous in $z$.

From (3.5) and the Taylor expansion it follows that $\left(\partial^{2} h(\mu)>0, z \leq 1\right)$

$$
u_{g}\left(q+e_{0}\right)-u_{g}\left(e_{0}\right) \leq \tilde{C}, \quad|q| \leq 1, \quad \tilde{C}=\max _{0 \leq z \leq z^{0}} \sum_{s=2}^{2 n} z^{2 n-s n}\left|\partial^{s} h(\mu)\right|(s !)^{-1}
$$

Taylor expansion gives possibility to choose sufficiently small $\varepsilon$ such that 


$$
h(q+\mu)-h(\mu) \geq \theta q^{2}, \quad-\varepsilon \mu \leq q \leq \varepsilon \mu .
$$

$\mu_{j}$ are shallow minima with respect to the deepest minimum $\mu$, that is, $\mu_{j}$ and $h\left(\mu_{j}\right)$ tends to zero for vanishing $z$ ( $h$ is continuous). As a result for $\varepsilon<\frac{1}{2}$ (we assumed that $\left.\mu_{j} \leq \frac{1}{4} \mu\right)$

$$
\begin{gathered}
h(q)-h(\mu) \geq \theta_{*}, \quad q \in \mathbb{R}^{+} \backslash[(1-\varepsilon) \mu,(1+\varepsilon) \mu], \\
\theta_{*}=\min _{z \in\left[0, z^{0}\right]}\{[h((1+\varepsilon) \mu)-h(\mu), h((1-\varepsilon) \mu)-h(\mu)\}>0 .
\end{gathered}
$$

Lemma 3.1. Let $e_{+}=z^{-1} \mu, \mu$ be the deepest minima of $u, h$, respectively. Then there exitsts a positive constant $\kappa^{-}$such that

$$
\kappa^{-} z^{-(2 l-n+1)} \leq \int q^{2 l} \exp \left\{-\beta\left[u(q)-u\left(e_{+}\right)\right]\right\} d q,
$$

and there exist positive constants $\kappa^{+}$and $g_{0}$ such that for $g \geq g_{0}$ the following inequality holds:

$$
\begin{gathered}
\int q^{2 l} \exp \left\{-\beta\left[u(q)-u\left(e_{+}\right)-z^{-n} \tilde{Q}(z q)\right]\right\} d q \leq \\
\leq \kappa^{+} z^{-(2 l-n+1)} e^{\beta \tilde{a} z^{-n}\left(\varepsilon a_{1}+z^{k} a_{2}\right)}, \quad \varepsilon>0,
\end{gathered}
$$

where

$$
\begin{gathered}
\tilde{Q}(q)=a_{1} Q^{0}(q-\mu ; \mu)+a_{2} z^{k} v^{0}(q-\mu), \\
\tilde{a}=Q^{0}(\mu ; \mu)+v^{0}(3 \mu), \quad k>0, \quad a_{s} \geq 0,
\end{gathered}
$$

the integration is performed over $\mathbb{R}$ and $\mu$ is given by (3.4).

Proof. From the definition of $h$ we derive the following formula:

$$
\int q^{2 l} e^{-\beta\left[u(q)-z^{-n} \tilde{Q}(z q)\right]} d q=z^{-1-2 l} \int q^{2 l} e^{-\beta z^{-2 n}\left[h(q)-z^{n} \tilde{Q}(q)\right]} d q .
$$

Here we rescaled the variable in the first integral by $z$.

Let $0 \leq \varepsilon<1$ and $R$ be such that

$$
h(q)-h(\mu)-\tilde{Q}(q) \geq q^{2}, \quad q \geq R \geq \mu .
$$

Let's decompose the positive half-line into three sets (the first set is in the round brackets)

$$
\mathbb{R}^{+}=([0,(1-\varepsilon) \mu] \cup[(1+\varepsilon) \mu, R]) \cup[(1-\varepsilon) \mu,(1+\varepsilon) \mu] \cup[R, \infty]
$$

change sign of $q$ in the integral in (3.7) and make estimates of the integral over these sets. For the integral over the second set we have

$$
\int_{(1-\varepsilon) \mu}^{(1+\varepsilon) \mu} q^{2 l} e^{-\beta z^{-2 n}\left[h(q)-z^{n} \tilde{Q}(q)\right]} d q \leq
$$




$$
\begin{gathered}
\leq(\mu(1+\varepsilon))^{2 l} \int_{-\varepsilon \mu}^{\varepsilon \mu} e^{-\beta z^{-2 n}\left[h(q+\mu)-z^{n} \tilde{Q}(q+\mu)\right]} d q \leq \\
\leq(\mu(1+\varepsilon))^{2 l} e^{\beta \tilde{a} z^{-n}\left(\varepsilon a_{1}+z^{k} a_{2}\right)} \int_{-\varepsilon \mu}^{\varepsilon \mu} e^{-\beta z^{-2 n} h(q+\mu)} d q .
\end{gathered}
$$

Here we applied the inequalities $Q^{0}( \pm \varepsilon \mu ; \mu) \leq \varepsilon^{n_{1}+n_{2}} Q^{0}(\mu ; \mu), \quad \varepsilon^{n_{1}+n_{2}} \leq \varepsilon$, $v^{0}( \pm \varepsilon \mu) \leq v^{0}(3 \mu)$. (3.5c) implies that the above integral in the right-hand side of (3.8) is less than

$$
\begin{gathered}
\int_{-\varepsilon \mu}^{\varepsilon \mu} e^{-\beta z^{-2 n} h(q+\mu)} d q \leq e^{-\beta z^{-2 n} h(\mu)} \int_{-\varepsilon \mu}^{\varepsilon \mu} e^{-\beta \theta z^{-2 n} q^{2}} d q \leq \\
\leq e^{-\beta z^{-2 n} h(\mu)} \int e^{-\beta \theta z^{-2 n} q^{2}} d q=\left(\pi^{-1} \beta \theta\right)^{-\frac{1}{2}} z^{n} e^{-\beta z^{-2 n} h(\mu)} .
\end{gathered}
$$

Inequality (3.5e) implies that the integral in (3.7) over the first set is less than

$$
R^{2 l+1} e^{-\beta z^{-2 n} h(\mu)} e^{-\beta z^{-2 n}\left[\theta_{*}-z^{n} \tilde{Q}(R)\right]} .
$$

For the integral over the third set we have

$$
\begin{gathered}
\int_{R}^{\infty} q^{2 l} e^{-\beta z^{-2 n}\left(h(q)-z^{n} \tilde{Q}\right)} d q \leq e^{-\beta z^{-2 n} h(\mu)} \int q^{2 l} e^{-\beta z^{-2 n} q^{2}} d q= \\
=\left(\beta^{-\frac{1}{2}} z^{n}\right)^{1+2 l} \int q^{2 l} e^{-q^{2}} d q .
\end{gathered}
$$

Combining (3.7) - (3.10) we obtain the right-hand side of (3.6a) with sufficiently small $\varepsilon$ (and arbitrary $\varepsilon$ )

$$
\kappa^{+}=(\bar{\mu}(1+\varepsilon))^{2 l}\left(\pi^{-1} \beta \theta\right)^{-\frac{1}{2}}+R^{2 l+1} \kappa^{\prime}+\beta^{-\frac{1+2 l}{2}} \int q^{2 l} e^{-q^{2}} d q, \quad \bar{\mu}=\max _{z \in\left[0, z^{0}\right]} \mu
$$

where $\kappa^{\prime}=\sup _{z \in\left[0, z^{0}\right], \theta_{*}-z^{n} \tilde{Q}(R) \geq \varepsilon_{0}} z^{-n+1} e^{-\beta z^{-2 n} \varepsilon_{0}}<\infty$ and $g_{0}=\left\{g: \theta_{*}-\right.$ $\left.-z^{n} Q(R)=\varepsilon_{0}>0\right\}$.

Now we have to prove (3.6). Let's rescale the integral by $z^{n-1}$ (the new variable is $\left.z^{1-n} q\right)$

$$
\int q^{2 l} e^{-\beta u(q)} d q=z^{(n-1)(2 l+1)} \int q^{2 l} e^{-\beta u_{g}(q)} d q .
$$

Let $e_{0}$ be the deepest minimum of $u_{g}$ then

$$
\begin{gathered}
\int q^{2 l} e^{-\beta u_{g}(q)} d q \geq \int_{e_{0}-1}^{e_{0}+1} q^{2 l} e^{-\beta u_{g}(q)} d q=\int_{-1}^{1}\left(q+e_{0}\right)^{2 l} e^{-\beta u_{g}\left(q+e_{0}\right)} d q \geq \\
\geq\left(e_{0}-1\right)^{2 l} e^{-\beta z^{-2 n} h(\mu)} \int_{-1}^{1} e^{-\beta\left(u_{g}\left(q+e_{0}\right)-u_{g}\left(e_{0}\right)\right)} d q .
\end{gathered}
$$


Substituting (3.5b) into the last inequality we obtain

$$
\int q^{2 l} e^{-\beta u_{g}(q)} d q \geq\left(e_{0}-1\right)^{2 l} e^{-\beta z^{-2 n} h(\mu)} e^{\tilde{C}} .
$$

From (3.11), (3.1), (3.3) we deduce that

$$
\int q^{2 l} e^{-\beta u_{g}(q)} d q \geq z^{-2 n l}\left(\mu-z^{n}\right)^{2 l} e^{-\beta z^{-2 n} h(\mu)} e^{-\beta \tilde{C}}=z^{-2 n l} \kappa^{-} e^{-\beta z^{-2 n} h(\mu)}
$$

where $\kappa^{-}=\frac{\mu_{0}}{4} e^{-\beta \tilde{C}}$. Formulae (3.10a) and (3.12) prove (3.6) for the integral over the positive real line. The same inequality holds for integral over the negative real. This follows easily by changing the sign of $q$ in the integral. The bounds for $\tilde{Q}(-q)$ are the same for $\tilde{Q}$ (since $Q^{0}(q-\mu ; \mu)$ is even $\left.i q\right)$. This concludes the proof of the lemma.

Now we can derive the bound for the integral in (2.12).

Proposition 3.1. For $g \geq g_{0}$ there exist positive constants $\kappa, \kappa^{\prime}$ such that the following inequality is valid:

$$
I_{*}\left(g, Q^{0}\right) \leq \kappa e^{\beta e_{0} \varepsilon_{*}}, \quad \int q^{2 l} e^{-\beta u_{*}^{+}(q)} d q \leq \kappa^{\prime} z^{-2 l n}, \quad \varepsilon>0 .
$$

Proof. From the definition of $h$ we see that $u_{g}\left(z^{-n} q\right)=z^{-2 n} h(q)$. So after the rescaling of the variable by $z^{-n}$ in the integral $I_{*}(g, Q)$, taking into account that $\epsilon=$ $=z^{2 n(n-1)}$,

$$
\epsilon v^{0}\left(z^{-n} q\right) \leq v^{0}(q), \quad e_{0}^{-2\left(n_{1}+n_{2}\right)+1} Q^{0}\left(z^{-n} q ; e_{0}\right)=z^{-n} \mu^{-2\left(n_{1}+n_{2}\right)+1} Q^{0}(q ; \mu)
$$

we obtain

$$
I_{*}(g, Q) \leq z^{-n} e^{\beta\left(\zeta_{0}+1\right)} \int \exp \left\{-\beta z^{-2 n}\left[h(q+\mu)-h(\mu)-z^{n} \tilde{Q}_{\mu}(q)\right]\right\} d q,
$$

where

$$
\tilde{Q}_{\mu}(q)=\mu^{-2\left(n_{1}+n_{2}\right)+1} Q^{0}(q ; \mu)+(\zeta+1) z^{n} v^{0}(q) .
$$

Formulae (3.14), (3.7), (3.6a) prove the inequality for $I_{*}\left(g, Q^{0}\right)$, in which $\kappa=e^{\beta\left(\zeta_{0}+1\right)} \kappa^{+}, \varepsilon_{*}=2 \tilde{a} \varepsilon \mu^{0}, a_{1}=\mu^{-2\left(n_{1}+n_{2}\right)}, a_{2}=1+\zeta, k=n$, since in this case $\tilde{Q}_{\mu}(q-\mu)=\tilde{Q}(q)$.

By the same argument one derives

$$
\begin{gathered}
\int q^{2 l} e^{-\beta u_{*}^{+}(q)} d q \leq \\
\leq z^{-n(2 l+1)} e^{\beta\left(\zeta_{0}+1\right)} \int q^{2 l} \exp \left\{-\beta z^{-2 n}\left[h(q+\mu)-h(\mu)-z^{n} \tilde{Q}_{\mu}(q)\right]\right\} d q .
\end{gathered}
$$

Translating $q$ by $-\mu$ and applying (3.7) and (3.6a) we obtain the needed bound with $\kappa^{\prime}=\kappa(1+\mu)^{2 l}$.

Proof of Theorem 2.1. In obtaining bounds for integrals in (2.3), (2.4) we rely on (1.5) from which it follows that:

$$
u\left(q_{1}, q_{2}\right)=u\left(q_{1}\right)+u\left(q_{2}\right)+g\left(q_{x}-q_{y}\right)^{2} Q\left(q_{x}, q_{y}\right) .
$$

ISSN 1027-3190. Укр. мат. журн., 2006, 58, № 3 
Let's rescale the variables in the integrals by $z^{n-1}$. As a result

$$
\begin{gathered}
\left\langle\sigma^{2 n_{0}}+\sigma^{\prime 2 n_{0}}\right\rangle=z^{2 n_{0}(n-1)} Z_{g}^{-1}(2) \int\left(q_{1}^{2 n_{0}}+q_{2}^{2 n_{0}}\right) e^{-\beta u_{g}\left(q_{1}, q_{2}\right)} d q_{1} d q_{2}, \\
Z_{g}(2)=\int e^{-\beta u_{g}\left(q_{1}, q_{2}\right)} d q_{1} d q_{2}=z^{-2(n-1)} Z(2), \\
u_{g}\left(q_{1}, q_{2}\right)=u_{g}\left(q_{1}\right)+u_{g}\left(q_{2}\right)-2 u_{g}\left(e_{0}\right)+g z^{2 n_{0}(n-1)}\left(q_{x}-q_{y}\right)^{2} Q\left(q_{x}, q_{y}\right) .
\end{gathered}
$$

The following inequality is true:

$$
\left(q_{1}-q_{2}\right)^{2} Q\left(q_{1}, q_{2}\right) \leq z^{-2\left(n_{0}-1\right) n} \bar{Q}, \quad q_{j} \in\left[e_{0}-1, e_{0}+1\right],
$$

where

$$
\bar{Q}=4 \max _{0 \leq z \leq 1} Q\left(1+z^{n} \mu, 1+z^{n} \mu\right) .
$$

The last inequality yields $\left(g=z^{-2\left(n-n_{0}\right)}\right)$

$$
u_{g}\left(q_{1}, q_{2}\right) \leq u_{g}\left(q_{1}\right)+u_{g}\left(q_{2}\right)-2 u_{g}\left(e_{0}\right)+\bar{Q}, \quad q_{j} \in\left[e_{0}-1, e_{0}+1\right] .
$$

As a result (see the derivation of (3.11))

$$
\begin{gathered}
Z_{g}(2) \geq \int_{e_{0}-1}^{e_{0}+1} \int_{e_{0}-1}^{e_{0}+1} e^{-\beta u_{g}\left(q_{1}, q_{2}\right)} d q_{1} d q_{2} \geq \\
\geq e^{-\beta \bar{Q}}\left(\int_{e_{0}-1}^{e_{0}+1} e^{-\beta\left(u_{g}(q)-u_{g}\left(e_{0}\right)\right)} d q\right)^{2} \geq e^{-\beta(\bar{Q}-\bar{C})} .
\end{gathered}
$$

The same argument leads to

$$
\begin{gathered}
\int\left(q_{1}^{2 n_{0}}+q_{2}^{2 n_{0}}\right) e^{-\beta u_{g}\left(q_{1}, q_{2}\right)} d q_{1} d q_{2} \geq \\
\geq\left(e_{0}+1\right)^{2 n_{0}} \int_{e_{0}-1}^{e_{0}+1} \int_{e_{0}-1}^{e_{0}+1} e^{-\beta u_{g}\left(q_{1}, q_{2}\right)} d q_{1} d q_{2} \geq \\
\geq 2\left(e_{0}+1\right)^{2 n_{0}} e^{-\beta \bar{Q}}\left(\int_{e_{0}-1}^{e_{0}+1} e^{-\beta\left(u_{g}(q)-u_{g}\left(e_{0}\right)\right)} d q\right)^{2} \geq \\
\geq 2\left(e_{0}+1\right)^{2 n_{0}} e^{-\beta(\bar{Q}-\bar{C})} .
\end{gathered}
$$

From (3.19) and right-hand side of (3.6a) for $l=0$, we see that for sufficiently small $z$ there exists the constant $C_{+}$such that

$$
\begin{gathered}
\left\langle\sigma^{2 n_{0}}+{\sigma^{\prime}}^{2 n_{0}}\right\rangle \geq \\
\geq\left(\kappa^{+}\right)^{-1} z^{-2(n-1)} z^{2 n_{0}(n-1)} z^{2(n-1)} z^{-2 n_{0} n}\left(\mu+z^{n}\right)^{2 n_{0}} e^{-\beta(\bar{Q}-\bar{C})} \geq z^{-2 n_{0}} C_{+} .
\end{gathered}
$$


Hence, (2.5) is proven since $-2 n_{0}=2\left(n-n_{0}\right)-2 n$ and $z^{2\left(n-n_{0}\right)}=g^{-1}$.

To prove (2.6) it is sufficient now to use the bound

$$
\begin{gathered}
\left\langle-\sigma^{2 n_{0}}-{\sigma^{\prime}}^{2 n_{0}}+\sigma^{k}{\sigma^{\prime}}^{l}+{\sigma^{\prime}}^{k} \sigma^{l}\right\rangle=\left\langle\left(\sigma-\sigma^{\prime}\right)^{2} Q\left(\sigma, \sigma^{\prime}\right)\right\rangle \leq \\
\leq z^{2 n_{0}(n-1)} Z_{g}^{-1}(2) \int\left(q_{1}-q_{1}\right)^{2} Q\left(q_{1}, q_{2}\right) e^{-\beta u_{g}\left(q_{1}, q_{2}\right)} d q_{1} d q_{2} \leq \\
\leq(g \beta)^{-1} \kappa_{1} \frac{Z_{g}^{0}(2)}{Z_{g}(2)},
\end{gathered}
$$

where

$$
\kappa_{1}=\max _{q \geq 0} q e^{-q}, \quad Z_{g}^{0}(2)=\left(\int e^{-\beta\left(u_{g}(q)-u_{g}\left(e_{0}\right)\right)} d q\right)^{2} .
$$

From (3.6a) for $l=0, \tilde{Q}=0,(3.1)$ (one has to take into account the equality $u\left(e_{+}\right)=$ $=u_{g}\left(e_{0}\right)=z^{-2 n} h(\mu)$, rescale the variable in the integral by $z^{n-1}$ ) and (3.18) it follows that $Z_{g}^{0}(2) \leq\left(\kappa^{+}\right)^{2}$. This together with (3.18) yields (2.6) with $C_{-}=\kappa_{1}\left(\kappa^{+}\right)^{2} e^{-\beta(\bar{Q}-\bar{C})}$. This concludes the proof of Theorem 1.2.

4. Basic superstability bound. A measurable function $U\left(q_{X}\right)$ is required to satisfy the superstability and regularity conditions

$$
\begin{gathered}
U\left(q_{X}\right)-U^{+}\left(q_{X}\right) \geq \sum_{x \in X} u^{-}\left(q_{x}\right), \\
\left|W\left(q_{X_{1}} ; q_{X_{2}}\right)\right| \leq \frac{1}{2} \sum_{x \in X_{1}, y \in X_{2}} \Psi(|x-y|)\left[v\left(q_{x}\right)+v\left(q_{y}\right)\right], \\
X_{1} \cap X_{2}=\varnothing, \quad v \geq 0
\end{gathered}
$$

where

$$
U^{+}\left(q_{X}\right)=\sum_{x, y \in X} \varphi_{x, y}^{+}\left(q_{x}, q_{y}\right), \quad \varphi^{+} \geq 0,
$$

and all the functions are measurable. The following integrals are necessary attributes of the superstability bound since its main parameter $c$ depends on them

$$
\begin{gathered}
\bar{u}(q)=u(q)+\|\Psi\|_{1} v(q), \quad I_{r}=e^{-\frac{1}{2} \beta\|\Psi\|_{1} \bar{v}_{r}} I_{0}, \quad I_{0}=\int_{|q| \leq r} e^{-\beta \bar{u}(q)} d q, \\
I(\varepsilon)=\int \exp \left\{-\beta\left[u^{-}(q)-3 \varepsilon v(q)\right]\right\} d q, \quad \bar{v}_{r}=\sup _{|q| \leq r} v(q) .
\end{gathered}
$$

Theorem 4.1. Let $\rho^{\Lambda}\left(q_{X}\right)$ be the correlation functions corresponding to the potential energy $U\left(q_{\Lambda}\right)$ which satisfies (4.1), (4.2). Let's put $\psi(x)=|x|^{k}, l_{j}=(1+2 \alpha)^{j}$ and require that

$$
\|\psi \Psi\|_{1} \leq \infty, \quad\|\Psi\|_{1}\left[(1+3 \alpha)^{2(d+k)}-1\right] \leq \frac{\varepsilon}{2}
$$

where $0<3 \varepsilon<1,\|\Psi\|_{p}=\sum_{x}|\Psi(|x|)|^{p}$ and the summation is performed over $\mathbb{Z}^{d}$. Then the following superstability bound is valid:

ISSN 1027-3190. Укр. мат. журн., 2006, 58, № 3 


$$
\rho^{\Lambda}\left(q_{X}\right) \leq \exp \left\{-\beta\left[U^{+}\left(q_{X}\right)+\sum_{x \in X}\left(u^{-}\left(q_{x}\right)-3 \varepsilon v\left(q_{x}\right)\right)\right]+|X| c\left(\varepsilon, I_{r}^{-1}, I(\varepsilon)\right)\right\}
$$

and there exist the positive numbers $c^{0}, \xi$ such that for $V_{j}=\left(1+2 l_{j}\right)^{d}$ the following representation is true:

$$
c\left(\varepsilon, z^{\prime}, z\right)=c^{0}+\ln \left(1+\xi z^{\prime}+f\left(\varepsilon, z z^{\prime}\right)\right), \quad f(\varepsilon, z)=\sum_{j \geq 0} e^{-\varepsilon \psi\left(l_{j}\right) V_{j}} z^{V_{j}} .
$$

Moreover, if $\Psi$ has a finite support then $c^{0}$ does not depend on $\varepsilon$ and $\xi$ is bounded in $\varepsilon$.

The bound (4.3) differs from the Ruelle bound by the presence of $e^{-\beta U^{+}}$, more general $\psi$ and the condition for $\alpha$ ( $k=1$ in the Ruelle condition) and, also, less general $l_{j}$.

Our $\psi$ enables to control an asymptotics of $c$ in $\varepsilon$ needed for providing the right asymptotics of $e_{*}(g)$ in $g$ in Theorem 2.2 after putting $\varepsilon=z^{2(n-1) n}=\left(\mu^{-1} e_{0}\right)^{-2(n-1)}$. The character of the asymptotics of $f$ and $c$ is depicted in the following corollary proven in the appendix.

Corollary 4.1. Let the conditions of Theorem 4.1 be satisfied and $k \geq d$.

(a) Then the following inequality is true:

$$
\lim _{\varepsilon \rightarrow 0} \varepsilon^{\frac{2 d}{k+d}} \ln f(\varepsilon, z) \leq 2^{d \frac{2 k+d}{k+d}}(\ln z)^{2} .
$$

(b) Moreover, if $\Psi$ has a finite support, then the following inequality holds:

$$
\lim _{\varepsilon \rightarrow 0} \varepsilon^{\frac{2 d}{k+d}} c\left(\varepsilon, z, z^{\prime}\right) \leq 2^{d \frac{2 k+d}{k+d}}\left(\ln z z^{\prime}\right)^{2} .
$$

Assertion (b) follows from (a) and and the last statement of Theorem 4.1.

The next lemma reduces proof of Theorem 2.2 to a determination of the asymptotics of $c$ in $\varepsilon=z^{2 n(n-1)}$ for fixed $z, z^{\prime}$.

Lemma 4.2. Let $u_{*}^{-}, v^{0}$ be the functions in the superstability and regularity conditions (1.6), (1.7). Let, also, the integrals $I\left(z^{2 n(n-1)}\right), I_{r}\left(z^{2 n(n-1)}\right)$, associated to them (instead of $\left.u^{-}, v\right)$ be denoted by $I_{*}(g), I_{* r}(g)$, respectively. Then the functions $\left(I_{* r}\right)^{-1}(g), I_{*}(g)$, are bounded in $g$.

Proof. The integral $I_{*}(g)$ is bounded due to Proposition 3.1. All the functions in the expressions for $I_{* r}(g)$ tend to a finite limit in $g$ at infinity. Indeed, $u_{*}$ tends to a finite limit due to (3.5a). For the inverse power of this integral the Jensen inequality is used and the Lebesque dominated convergence theorem is easily applied since the integration is performed over a compact space. This concludes the proof of the lemma.

For short-range interaction $k$ in Corollary 4.1 may be arbitrary. Thus, Lemma 4.1 and Corollary 4.1 show that $e_{*}$ satisfies the property indicated in Theorem 2.2.

Corollary 4.2. Let the conditions of Theorem 2.2 be satisfied and $c$ be given in Theorem 4.1. Then the function $e_{*}$ from Theorem 2.2 is given by

$$
e_{*}(g)=c\left(z^{2 n(n-1)}, I_{* r}(g), I_{*}(g)\right)
$$

and behave as $e_{0}^{\theta}$ asymptotically in $g$ at infinity where $\theta$ is an arbitrary small number.

This corollary completes the proof of Theorem 2.2.

Proof of Corollary 4.1 can be found in [5]. 
Appendix A. To prove that $\phi_{*}$ satisfies (1.7) one has to use the inequalities $(k, l$ are positive integers, $q, q^{\prime} \geq 0$ )

$$
\left(q \pm q^{\prime}\right)^{l} \leq 2^{l}\left(q^{l}+q^{\prime l}\right), \quad q^{k} q^{\prime l}+q^{l} q^{\prime k} \leq 2^{k+l}\left(q^{k+l}+q^{\prime k+l}\right) .
$$

The second inequality and the equality $Q^{\prime}(1,1)=1$ lead to

$$
Q^{\prime}\left(q, q^{\prime}\right) \leq 2^{2 n_{2}}\left(q^{2 n_{2}}+q^{2 n_{2}}\right) .
$$

As a result of the first previous and last inequalities one obtains for $r=c z^{-1}, c>0$, $z \leq 1$

$$
\begin{gathered}
2^{4 n_{2}}\left(q-q^{\prime}\right)^{2 n_{1}}\left(q^{2 n_{2}}+q^{2 n_{2}}+2 r^{2 n_{2}}\right) \leq \\
\leq 2^{4 n_{2}+2 n_{1}}\left(q^{2 n_{1}}+q^{2 n_{1}}\right)\left(q^{2 n_{2}}+q^{\prime 2 n_{2}}+2 r^{2 n_{2}}\right) \leq \\
\leq 2^{4 n_{2}+2 n_{1}}\left[q^{2 n_{2}+2 n_{1}}+q^{2 n_{2}+2 n_{1}}+q^{2 n_{1}} q^{2 n_{2}}+\right. \\
\left.+q^{2 n_{2}} q^{\prime 2 n_{1}}+2\left(q^{2 n_{1}}+q^{\prime 2 n_{1}}\right) r^{2 n_{2}}\right] \leq \\
\leq 2^{4 n_{2}+2 n_{1}}\left[\left(1+2^{2 n_{1}+2 n_{2}}\right)\left(q^{2 n_{2}+2 n_{1}}+q^{2 n_{2}+2 n_{1}}\right)+2\left(q^{2 n_{1}}+q^{\prime 2 n_{1}}\right) r^{2 n_{2}}\right] \leq \\
\leq 2^{4 n_{2}+2 n_{1}}\left(1+2^{2 n_{1}+2 n_{2}}\right)\left(1+c^{2 n_{2}}\right) z^{-2 n_{2}+2(n-1) n_{1}} \times \\
\times\left[v^{0}\left(z^{-(n-1)} q\right)+v^{0}\left(z^{-(n-1)} q^{\prime}\right)\right]
\end{gathered}
$$

From the equality $e_{0}=\mu z^{-n}$ (see the third section) it follows that $(\mu=c)$

$$
\begin{gathered}
\left|\phi_{*}\left(q, q^{\prime}\right)\right|=\left|\phi_{g}\left(q+e_{0}, q^{\prime}+e_{0}\right)\right| \leq \\
\leq 2^{4 n_{2}+2 n_{1}}\left(1+2^{2 n_{1}+2 n_{2}}\right)\left(1+\mu^{2 n_{2}}\right) z^{-2 n_{2}+2(n-1) n_{1}}\left[v^{0}(q)+v^{0}\left(q^{\prime}\right)\right] .
\end{gathered}
$$

The derivation of the regularity condition (1.7), now, is straightforward since $\mu$ is bonded in $z \leq 1$.

Appendix B. Let's put

$$
\begin{gathered}
u^{1}(q)=\eta_{2 l} q^{2 l}, \quad h^{1}(q)=\eta_{2 l} z^{2(n-l)} q^{2 l}, \\
2 n-1=2 s+2 l-1, \quad 2 n_{0}-1=s+2 l-1, \quad s \in \mathbb{Z}^{+} .
\end{gathered}
$$

In this case root equation is given by

$$
2^{-1} \partial h(q)=n \eta q^{2 n-1}-n_{0} q^{2 n_{0}-1}+l \eta_{2 l} z^{2(n-l)} q^{2 l-1}=0,
$$

or

$$
\begin{gathered}
q^{2 l-1}\left(n \eta q^{2 s}-n_{0} q^{s}+l \eta_{2 l} z^{2(n-l)}\right)=0, \\
q=0, \quad n \eta x^{2}-n_{0} x+l \eta_{2 l} z^{2(n-l)}=0, \quad x=q^{s} .
\end{gathered}
$$

The nontrivial roots of this quadratic equation are given by

$$
2^{-\frac{1}{s}}\left(\mu^{0} \pm \mu^{\prime}\right)^{\frac{1}{s}}, \quad \mu^{\prime}=\left(\mu^{0}-\frac{4 l}{n \eta} z^{2(n-l)} \eta_{2 l}\right)^{\frac{1}{2}}
$$

ISSN 1027-3190. Укр. мат. журн., 2006, 58, № 3 
If $\eta_{2 l}<0$ then there is only one non-zero root

$$
\mu=2^{-\frac{1}{s}}\left(\mu^{0}+\mu^{\prime}\right)^{\frac{1}{s}}, \quad \mu^{\prime}=\left(\mu^{0}+\frac{4 l}{n \eta} z^{2(n-l)}\left|\eta_{2 l}\right|\right)^{\frac{1}{2}} .
$$

If $\eta_{2 l}>0$ then there are two non-zero roots $\mu, \mu^{-}$for sufficiently small $z$

$$
\begin{gathered}
\mu=2^{-\frac{1}{s}}\left(\mu^{0}+\mu^{\prime}\right)^{\frac{1}{s}}, \quad \mu^{\prime}=\left(\mu^{0}-\frac{4 l}{n \eta} z^{2(n-l)}\left|\eta_{2 l}\right|\right)^{\frac{1}{2}}, \\
\mu^{-}=2^{-\frac{1}{s}}\left(\mu^{0}-\mu^{\prime}\right)^{\frac{1}{s}}=z^{\frac{2(n-l)}{s}}(\tilde{\mu}(z))^{\frac{1}{s}}, \quad \tilde{\mu}(0)>0 .
\end{gathered}
$$

For $\mu, \mu^{-}$the expansions (3.4), (3.5) are valid, respectively. The trivial root has multiplicity $2 l-1$, the root $\mu^{-}$is simple and other roots are complex.

Appendix C. Let's consider the polynomial

$$
x^{2 n}+y^{2 n}-x^{k} y^{2 n-k}-y^{k} x^{2 n-k} .
$$

If it is positive for positive $x, y$ then it is positive for all $x, y$. It is not difficult to check that for positive arguments the second derivative in $k$ of the polynomial is negative, that is, the polynomial is a convex function in $k$ on the interval $[0,2 n]$. Besides, It is has a root of the second order for the value $x=y$, i.e., the first and the second derivative are equal to zero for the value. This proves (1.5), i.e., existence of $Q$. These remarkably simple arguments were proposed by the referee. The initial proof was very lengthy but it was constructive and gave the explicit expression for $Q$. Since we do not use it in our subsequent bounds this proof was omitted by us.

Remark 1. Another method of proving of existence of lro for the classical ferromagnetic oscillator systems was proposed in [15]. In [16] the static quantum Holstein model was reduced to a classical linear oscillator system with a non-n-n nonpair interaction and for such the system occurrence of the antiferromagnetic lro was proven with a help of a special generalized Peierls argument. In [17], the classical n-n linear oscillator systems were treated in terms of the Pirogov - Sinai theory (see $[18,19])$ and most profound results concerning phase diagrams were obtained. These ideas were applied in the lattice Higgs gauge field models in [20].

Remark 2. The potential energy for ferromagnetic GKS systems is given as follows:

$$
\begin{gathered}
U^{\prime}\left(q_{\Lambda}\right)=\sum_{\left(A, n_{A}\right), A \subseteq \Lambda} J_{A, n_{A}} q_{[A]}^{n_{A}}, \quad q_{[A]}^{n_{A}}=\prod_{x \in A} q_{x}^{n_{x}}, \\
n_{A}=\left(n_{x}, x \in A\right), \quad n_{x} \in \mathbb{Z}^{+}, \\
J_{A, n_{A}} \geq 0, \quad \sum_{x \in A} n_{x}=2 n(A), \quad n_{x}, n(A) \in \mathbb{Z}^{+} .
\end{gathered}
$$

In the last line the intermediate condition implies that the potential energy is invariant under the change of the signs of all the oscillator variables.

In order to guarantee finiteness of the partition function and correlation function it has to be assumed that

$$
\sup _{A} n(A)<n, \quad \max _{x} \sum_{A, x \in A} J_{A, n_{A}}<\infty .
$$


Remark 3. In general case a depth of the potential in (1.5) is controlled by $n-1$ parameters, i.e., coefficients of the polynomial $u$. The different subsets of this space can be described with the help of one parameter $g$ and the representation

$$
u(q)=\eta q^{2 n}-\sum_{s=1}^{n-1} \eta_{s} g^{n_{s}} q^{2 s} .
$$

To deal with this case one has to find an appropriate rescaling reducing the polynomial to a sum of a polynomial with easily calculated minima and an additional polynomial depending on $g^{-1}$.

Remark 4. Asymptotics of the integral in (3.6) can be found with the help of the usual one-dimensional Laplace method [21]. It has to be generalized for finding asymptotics of the integral in (3.6a). Our arguments in the proof of (3.6a) are close to the arguments of the usual one-dimensional Laplace method but not identical to them. To find asymptotics of integrals in (2.5), (2.6) one has do employ the two-dimensional Laplace method. It is less known than its one-dimensional version. Our proofs are optimal and there is no need for a reader to check conditions in a general statement of the Laplace method for establishing asymptotics of our integrals.

1. Glimm J., Jaffe A., Spencer T. Phase transitions for $\varphi_{2}^{4}$ quantum fields // Communs Math. Phys. - 1975. 45. - P. 203-216.

2. Frohlich J., Lieb E. Phase transitions in anisotropic lattice spin systems // Ibid. - 1978. - 60. - P. 233 - 267.

3. Skrypnik W. LRO in lattice systems of linear oscillators with strong bilinear pair nearest-neighbour interaction // J. Phys. A. - 1999. - 32. - P. $7039-7048$.

4. Skrypnik W. I. LRO in lattice systems of linear classical and quantum oscillators. Strong nearest-neighbor pair quadratic interaction // J. Stat. Phys. - 2000. - 100, № 5/6. - P. 853-870.

5. Skrypnik W. I. Long-range order in non-equilibrium systems of interacting Brownian linear oscillators // Ibid. - 2002. - 111, № 1/2. - P. $291-321$.

6. Skrypnik W. I. Long-range order in linear ferromagnetic oscillator systems. Strong pair quadratic $\mathrm{n}-\mathrm{n}$ potential // Ukr. Math. J. - 2004. - 56, № 6. - P. 810-817.

7. Simon B. The $P(\phi)_{2}$ Euclidean (Quantum) field theory. - Princeton (New Jersey): Princeton Univ. Press, 1974.

8. Ginibre J. General formulation of Griffiths' inequalities // Communs Math. Phys. - 1970 - 16. - P. 310 328.

9. Kиnz H. Analiticity and clustering properties of unbounded spin systems // Ibid. - 1978. - 59. - P. 53-69.

10. Ruelle D. Probability estimates for continuous spin systems // Ibid. - 1976. - 50. - P. 189-194.

11. Bricmont J., Fontaine J.-R. Correlation inequalities and contour estimates // J. Stat. Phys. - 1981. - 26, № 4. - P. 745.

12. Ruelle D. Statistical Mechanics. Rigorous results. - W. A. Benjamin Inc., 1969. - 219 p.

13. Benfatto G., Marchioro C., Presutti E., Pulvirenti M. Superstability estimates for anharmonic systems // J. Stat. Phys. - 1980. - 22, № 3. - P. 349-359.

14. Reed M., Simon B. Methods of modern mathematical physics. - New York etc.: Acad. Press, 1976 (Rus. trans.: Moscow: Mir, 1976. -395 p.).

15. Kunz H., Payandeh B. Existence of a phase transition for a class of ferroelectric models near the displacive limit // Phys. Rev. B. - 1978. - 18. - P. 2276-2280.

16. Lebowitz J., Macris $N$. Low-temperature phases of itinerant fermions interacting with classical phonons: The static Holstein model // J. Stat. Phys. - 1994. - 76, № 1/2. - P. 91 - 123.

17. Borgs $C$., Waxler R. First order phase transitions in unbounded spin systems. I: Construction of the phase diagram // Communs Math. Phys. - 1989. - 126. - P. 291-324.

18. Shlosman S. Reflection positivity method in mathematical theory of first order phase transitions // Uspekhi Mat. Nauk. - 1986. - 41, № 3. - P. 69 (in Russian).

19. Sinai Ya. G. Theory of phase transitions. Rigorous results. - Moscow: Nauka, 1980. -207 p.

20. Borgs C., Frohlich J. On the phase structure of the large n lattice Higgs model // Nucl. Phys. B. - 1989. 328. - P. $611-638$

21. Fedoryuk M. V. Asymptotics, integrals and series. - Moscow: Nauka, 1987. -544 p. 